\title{
Operational Performance and Status of the ATLAS Pixel Detector at the LHC
}

\author{
J. Jentzsch* \\ On behalf of the ATLAS Collaboration \\ CERN, \\ CH-1211 Geneve 23, Switzerland \\ *E-mail: jennifer.jentzsch@cern.ch
}

\begin{abstract}
The ATLAS Pixel Detector is the innermost detector of the ATLAS experiment at the Large Hadron Collider at CERN. The detector provides hermetic coverage with three cylindrical layers and three layers of pixel detectors located in forward and backward direction. It consists of approximately 80 million pixels that are individually read out via chips bump-bonded to $1744 \mathrm{n}^{+}$-inn silicon substrates. In this talk, results from the successful operation of the Pixel Detector at the LHC and its status after three years of operation will be presented, including monitoring, calibration procedures, timing optimization and detector performance. The record breaking instantaneous luminosities of $7.7 \cdot 10^{33} \mathrm{~cm}^{-2} \mathrm{~s}^{-1}$ recently surpassed at the Large Hadron Collider generate a rapidly increasing particle fluence in the ATLAS Pixel Detector. As the radiation dose accumulates, the first effects of radiation damage are now observable in the silicon sensors. A regular monitoring program has been conducted and reveals an increase in the silicon leakage current, which is found to be correlated with the rising radiation dose recorded by independent sensors within the inner detector volume. In the longer-term crystal defect formation in the silicon bulk is expected to alter the effective doping concentration, producing type-inversion and ultimately an increase of the voltage required to fully deplete the sensor. The fourth pixel layer at the radius of $3.5 \mathrm{~cm}$ will be added during the long shutdown 2013-2014 together with the replacement of pixel services.
\end{abstract}

Keywords: Large Hadron Collider (LHC); ATLAS; ATLAS Pixel.

\section{Introduction}

The ATLAS Pixel Detector ${ }^{1}$ is designed to ensure the tracking based on three hits for each particle traversing at a pseudo-rapidity up to $|\eta|<2.5$ in order to find primary and secondary vertices in the ATLAS ${ }^{2}$ experiment. In total 1744 modules, overlapping in $z$ and $r \varphi$ to reduce the inactive area fraction, have been installed. The detector was designed to withstand parti- 
cle radiation up to a fluence of $10^{15} 1 \mathrm{MeV} \mathrm{n}_{\mathrm{eq}} \mathrm{cm}^{-2}$ corresponding to a dose of $500 \mathrm{kGy}(50 \mathrm{Mrad}) . \mathrm{C}_{3} \mathrm{~F}_{8}$, set to $-20^{\circ} \mathrm{C}$, runs in cooling pipes integrated in the local support structures.

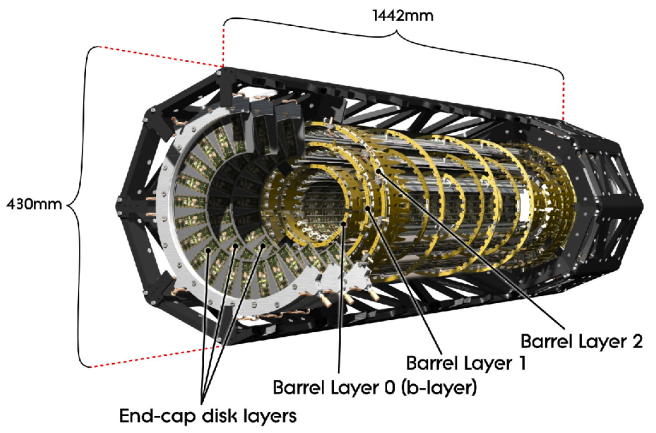

(a)

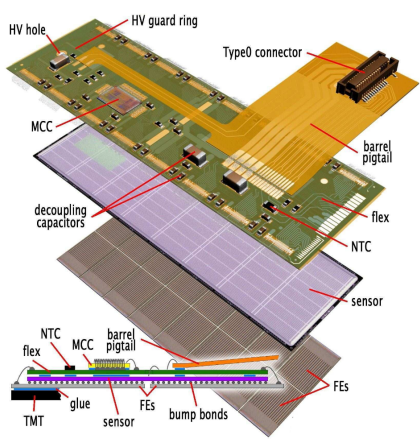

(b)

Fig. 1. (a) Perspective view of the ATLAS Pixel Detector and a (b) Pixel module ${ }^{1}$

The smallest unit of the Pixel Detector is a module (see fig. 1(b)). It consists of one sensor tile, $250 \mu \mathrm{m} \mathrm{n}^{+}$-in-n planar technology with a regular pixel size of $50 \times 400 \mu \mathrm{m}$. Since there are 16 readout chips connected to one sensor tile, the gaps between the FEs have been bridged with long pixels $(50 \times 600 \mu \mathrm{m})$ and ganged pixels (two sensor pixels connected to one read out pixel) respectively. Up to $600 \mathrm{~V}$ reverse bias voltage can be applied to the sensors. The amount of voltage needed to deplete the sensor depends mainly on the temperature and the irradiation level (see section 4). A Module Controller Chip on top of each module combines the FE information and builds module events which will then be read out. The different layers operate at $40 \mathrm{up}$ to $160 \mathrm{Mbit} / \mathrm{s}$ transmitting zero suppressed data.

\section{Calibration of the ATLAS Pixel Detector}

An internal injection and calibration circuit has been implemented to ensure a consistent response for all pixels. To effectively differentiate between noise and the actual signal a charge threshold can be set via a 7 bit DAC (TDAC). The discrimination digitalizes the analog sensor signal. In addition, the original amplitude is represented by the length of the output signal (time over threshold). This can be adjusted by tuning the feedback for each preamplifier using a 3 bit DAC (FDAC). The threshold and the inherent noise can be derived from the discriminator activation curve as a 
function of the internally injected charge. The current threshold setting for the ATLAS Pixel Detector is $3500 \mathrm{e}^{-}$with a typical dispersion of $40 \mathrm{e}^{-}$. For regular pixels the noise level is below $200 \mathrm{e}^{-}$, but naturally twice as high for ganged pixels.

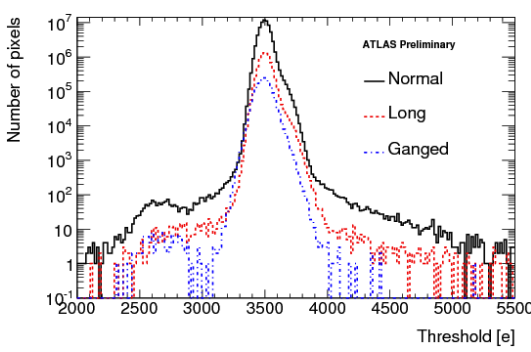

(a)

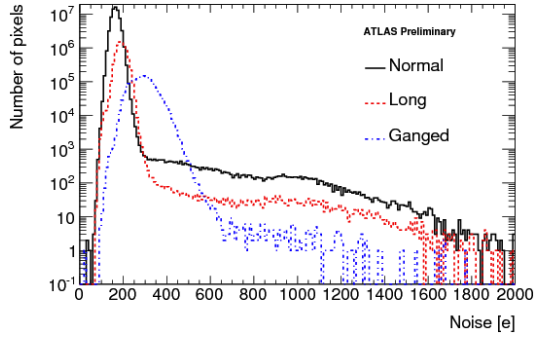

(b)

Fig. 2. (a) Operation threshold and (b) noise distributions of all ATLAS Pixel modules ${ }^{3}$

The time over threshold (ToT) is measured in units of bunch crossing, so in multiples of $25 \mathrm{~ns}$ in the case of the LHC. It is generally proportional to the injected or respectively collected charge. Since a MIP signal is expected to create a $20 \mathrm{ke}^{-}$signal in the sensor, the readout is tuned to provide a 30 bunch crossing long output signal at this reference charge. Because of the high ToT resolution the specific ionization $\mathrm{dE} / \mathrm{dx}$ of a track can be measured by the ATLAS Pixel Detector with a typical resolution of $10 \%$. It is even sufficient to distinguish a proton from a kaon in minimum bias events below $1 \mathrm{GeV}$.

\section{Performance Measurements}

The performance of a tracking detector highly depends on its hit to track association efficiency. It is well above $98 \%$ for the different layers of the ATLAS Pixel Detector (except for the outer disks) while the B-Layer efficiency is stated with $100 \%$ because of the track selection. In this computation dead modules are excluded, but otherwise dead regions and inefficiencies contribute. The rather low efficiency for the external discs is mainly due to inefficient regions in some modules caused by either defect front end chips or disconnected solder bump bonds.

In the presence of an electric and a magnetic field the charge carriers created by a passing particle within silicon drift along a direction at an angle with respect to the electric field direction. They usually spread over several pixels, depending on the angle of the incident particle. This spread 
is a minimum for an angle equal to the Lorentz angle. The measurement of the cluster sizes depending on the incident angle have been performed both, once with the solenoid off and once in operational magnetic field. The Lorentz angle is found to be consistent with 0 for no presence of a magnetic field. Using the data with magnetic field on the preliminary measurement of the Lorentz angle is $205 \pm 0.5 \mathrm{mrad}$ which is in the expectation of about $225 \mathrm{mrad}$.

Furthermore, the ToT information of each pixel is used for weighted cluster position determination, so called analog clustering, improving the spatial resolution mainly in the range of two-hit clusters in the transverse coordinate as well as in the longitudinal coordinate.

\section{Radiation Damages}

After collecting $29 \mathrm{fb}^{-1}$ of LHC data radiation damages are not negligible anymore. Non Ionizing Energy Losses (NIEL) are the cause for displacement defects in the sensors silicon bulk. The first measurable effect is the increase of leakage current through the bulk which is proportional to the fluence. Furthermore, the number of collected charges drops constantly and eventually, after a fluence of $0.3 \cdot 10^{14} 1 \mathrm{MeV} \mathrm{n}_{\mathrm{eq}} \mathrm{cm}^{-2}$ the effective doping concentration changes. This effect is called type inversion. The leakage currents, depletion voltages and depletion depths need to be constantly monitored and the applied voltages need to be adjusted accordingly to ensure an optimal data taking.

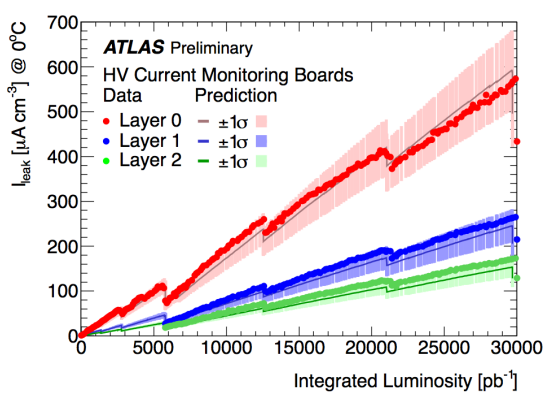

(a)

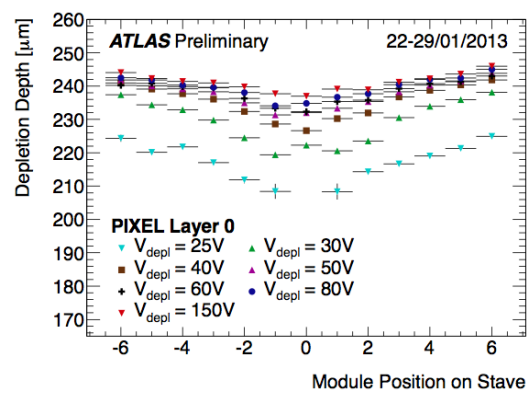

(b)

Fig. 3. (a) ATLAS Pixel leakage current trend and (b) track based B-Layer depletion voltage measurements ${ }^{3}$

The leakage current clearly follows the integrated luminosity (see fig. $3(\mathrm{a})$ ). The steps are consistent with annealing during detector warm up 
periods. The prediction is based on delivered luminosity, expected fluence from simulations and the "Dortmund model". Before irradiation, for instance before type inversion, the depletion voltage can be determined using the crosstalk method at different sensor bias voltages. Internally a very high charge (up to $200 \mathrm{ke}^{-}$) is injected into one pixel while the neighboring pixels are read out. Since the charge is very high it will talk over to the neighbor in case of an undepleted sensor. From performing these scans at different bias voltages one can calculate the effective depletion voltage.

After type inversion this method is not applicable anymore. However, actual tracks can be used to determine the depletion depth depending on the bias voltage. In fig. 3(b) results from the B-Layer are presented. It is already type inverted since it is the innermost layer and thus suffers most from radiation damages.

\section{Current status and plans for LS1}

At the end of Run1, 95\% of all modules were activated during data taking. 88 modules were disabled or problematic. The occurrences of the failures can mostly be linked to thermal cyclings and the failure modes indicate faults mainly in the services. Therefore, new Service Quarter Panels (nSQPs) are being mounted. They do not only replace the former services but they also route the opto-electrical transceivers to a more serviceable location. Thus, up to $92 \%$ of the failing modules can be recovered. The new services will also allow a later Layer 1 readout speed upgrade to $160 \mathrm{Mbit} / \mathrm{s}$. In addition, a new Diamond Beam Monitor for beam loss monitoring and bunch-bybunch luminosity measurements is being installed inside the nSQPs, $1 \mathrm{~m}$ from the interaction point. Furthermore, a fourth layer of pixel detectors, the Insertable B-Layer (IBL) ${ }^{4}$ hosting a new front end chip generation to meet the increased requirements, is being built and will be installed in 2014 .

\section{References}

1. G.Aad et al., JINST 3 (2008), P07007.

2. The ATLAS Collaboration, The ATLAS experiment at the Large Hadron Collider, JINST 3 (2008), S08003.

3. ATLAS Collaboration, 2013 https://twiki.cern.ch/twiki/bin/view/AtlasPublic/ApprovedPlotsPixel, https://twiki.cern.ch/twiki/bin/view/AtlasPublic/PixelPublicResults.

4. ATLAS IBL Collaboration, ATLAS Insertable B-Layer Technical Design Report, CERN-LHCC-2010-013, ATLAS-TDR-019, Addendum CERN-LHCC2012-009, ATLAS-TDR-019-ADD-1. 